\title{
Highly Diastereoselective and Enantioselective C-H Functionalization of 1,2-Dihydronaphthalenes: \\ A Combined C-H Activation-Cope Rearrangement Followed by a Retro-Cope Rearrangement
}

\author{
Huw M. L. Davies* and Qihui Jin \\ Department of Chemistry, University at Buffalo, The State University of New York, \\ Buffalo, NY 14260-3000
}

Supporting Information
A: General Procedure and Starting Materials (S2)
B: Procedures and data of all new compounds (S3-S17)
C: X-ray structure of compound B (S18-S19) 


\section{General Procedure:}

${ }^{1} \mathrm{H}$ NMR spectra were run at either 400 or $500 \mathrm{MHz}$, and ${ }^{13} \mathrm{C}$ NMR at either 75 or 125 $\mathrm{MHz}$ with the sample solvent being $\mathrm{CDCl}_{3}$ unless otherwise noted. Mass spectral determinations were carried out in GC-MS (EI), LC-MS (ESI) or by Instrument Center, Department of Chemistry, University at Buffallo. IR spectra were obtained using a Nicolet Impact series 420 FT-IR. Optical rotations were measured using a Jasco DIP-370 digital polarimeter. Elemental analyses were performed by Atlantic Microlabs Inc., Norcross GA. Enantiomeric excess was determined by HPLC using a Chiralcel OD-H or $(R, R)$-Whelk-O 1 chiral analytical column (UV detection at $254 \mathrm{~nm}$ ). Analytical TLC was performed on $0.25 \mathrm{~mm}$ E. Merck silica gel (60F-254) plates using UV light.

Glassware was dried in oven overnight then flame or heat-gun dried prior to use. Reactions were conducted under argon atmosphere. Column chromatography was carried out on Merck silica gel 60 (230-400 mesh). Solvent THF, Et ${ }_{2} \mathrm{O}, \mathrm{CH}_{3} \mathrm{CN}, \mathrm{CH}_{2} \mathrm{Cl}_{2}$ and toluene were dried by solvent purifier. 2,2-Dimethylbutane (DMB) was purified by passing through silica gel 60 (Merck, 230-400 mesh, activated by heating under argon), distilled from sodium then stored over MS ( $3 \AA$ ), and degassed (by bubbling argon gas for 5-10 min) prior to use.

\section{Starting Materials:}

The following starting materials $1,{ }^{1} 2,{ }^{2} 4,{ }^{3} 11,{ }^{4} 14 \mathbf{a}^{5}, 14 \mathrm{~b},{ }^{5} 17 \mathrm{~b},{ }^{6} 17 \mathbf{c},{ }^{6} 19^{7}$ and catalyst $\mathrm{Rh}_{2}(S \text {-DOSP })_{4}{ }^{8}$ were prepared according to the published procedure. Starting material 7 and $\mathbf{1 7}$ a were purchased from Aldrich $^{\circledR}$.

\footnotetext{
${ }^{1}$ Davies, H. M. L.; Clark, T. J. and Smith, H. D. J. Org. Chem. 1991, 56, 3817.

${ }^{2}$ Adamczyk, M.; Watt, D. A. and Netzel, D. A. J. Org. Chem. 1984, 49, 4226.

${ }^{3}$ Davies, H. M. L. and Jin, Q. Prod. Natl. Acad. Sci. USA 2004, 101, 5472.

${ }^{4}$ Maekawa, H.; Itoh, K.; Goda, S. and Nishiguchi, I. Chirality 2003, 15, 95.

${ }^{5}$ Harrowven, D. C. and Dainty, R. F. Tetrahedron, 1997, 53, 15771-15786.

${ }^{6}$ Magnus, P.; Lacour, J.; Coldham, I.; Mugrage, B. and Bauta, W. B. Tetrahedron 1995, 51, 11087.

7 (a) Kirkiacharian, B. S.; Danan, A. and Koutsourakis, P. G. Synthesis 1991, 879-881. (b)

Balasubramanian, T. and Balsubramanian, K. K. Tetrahedron Lett. 1991, 32, 6641-6644.

${ }^{8}$ Davies, H. M. L.; Bruzinski, P.; Hutcheson, D. K.; Kong, N. and Fall, M. J. J. Am. Chem. Soc. 1996, 118, 6897.
} 


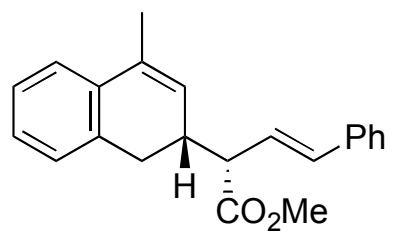

To a solution of 4-methyl-1,2-dihydronaphthalene $2(0.5 \mathrm{mmol})$ and $\mathrm{Rh}_{2}(S \text {-DOSP })_{4}(4.8$ $\mathrm{mg}, 0.0025 \mathrm{mmol})$ in 2,2-dimethylbutane $(1 \mathrm{~mL})$ was added a solution of $(E)$-methyl phenylvinyldiazoacetate $1(111 \mathrm{mg}, 0.55 \mathrm{mmol})$ in DMB $(5 \mathrm{~mL})$ over a $45 \mathrm{~min}$ period via syringe-pump at $0{ }^{\circ} \mathrm{C}$ (with crashed-ice cooling). The resulting mixture was then stirred overnight at $\mathrm{rt}(\mathrm{ca} .12 \mathrm{~h})$. The solvent was then evaporated under reduced pressure and the residue was purified by flash chromatography on silica gel (20:1 to 10:1 pentane/ether eluent) to provide 3 ( $151 \mathrm{mg}, 95 \%$ yield).

\section{$(S, 3 E)$-methyl 2-((R)-1,2-dihydro-4-methylnaphthalen-2-yl)-4-phenylbut-3-enoate}

\section{(3):}

white solid, mp $75-77^{\circ} \mathrm{C} ; \mathrm{R}_{\mathrm{f}} 0.35$ (10:1 pentane/ether); $[\alpha]_{\mathrm{D}}{ }^{25}-30.4$ (c 4.10, $\mathrm{CHCl}_{3}$ ); FTIR $\left(\mathrm{CH}_{2} \mathrm{Cl}_{2}\right)$ : 3059, 3026, 2949, 1733, 1490, 1450, 1435, 1260, 1193, 1158, $1027 \mathrm{~cm}^{-1}$; ${ }^{1} \mathrm{H}$ NMR $\left(500 \mathrm{MHz}, \mathrm{CDCl}_{3}\right) \delta$ 7.35-7.28 (m, 4H), 7.25-7.17 (m, 3H), $7.14(\mathrm{td}, J=7.0$, $1.8 \mathrm{~Hz}, 1 \mathrm{H}), 7.07$ (br d, $J=7.0 \mathrm{~Hz}, 1 \mathrm{H}), 6.41$ (d, $J=15.9 \mathrm{~Hz}, 1 \mathrm{H}), 6.14$ (dd, $J=15.9,9.8$ $\mathrm{Hz}, 1 \mathrm{H}$ ), 5.73 (br d, $J=3.0 \mathrm{~Hz}, 1 \mathrm{H}$ ), 3.68 (s, 3H), 3.10 (appt. t, $J=9.3 \mathrm{~Hz}, 1 \mathrm{H}$ ), 2.87 (dd, $J=14.2,6.0 \mathrm{~Hz}, 1 \mathrm{H}), 2.86-2.79(\mathrm{~m}, 1 \mathrm{H}), 2.71(\mathrm{dd}, J=14.2,6.5 \mathrm{~Hz}, 1 \mathrm{H}), 2.06(\mathrm{~s}, 3 \mathrm{H})$; ${ }^{13} \mathrm{C}$ NMR $\left(125 \mathrm{MHz}, \mathrm{CDCl}_{3}\right) \delta$ 173.6, 136.6, 135.2, 134.3, 133.61, 133.56, 128.5, 127.8, 127.7, 127.1, 126.5, 126.3, 126.2, 126.0, 123.0, 53.1, 51.8, 36.0, 31.6, 19.4; LC-MS (ESI) $\mathrm{m} / z$ (relative intensity): $319.2\left([\mathrm{M}+\mathrm{H}]^{+}, 100\right), 341.1\left([\mathrm{M}+\mathrm{Na}]^{+}, 0.4\right)$; HPLC analysis: $99.5 \%$ ee (Chiralcel OD-H, $2.0 \% i-\mathrm{PrOH}$ in hexane, $0.8 \mathrm{~mL} / \mathrm{min}, \lambda=254 \mathrm{~nm}, \mathrm{t}_{\mathrm{R}}=9.2$ min, major; $t_{R}=10.9$ min, minor); Anal. Calcd for $\mathrm{C}_{22} \mathrm{H}_{22} \mathrm{O}_{2}: \mathrm{C}, 82.99 ; \mathrm{H}, 6.96$. Found: C, $82.69 ; \mathrm{H}, 6.87$. 


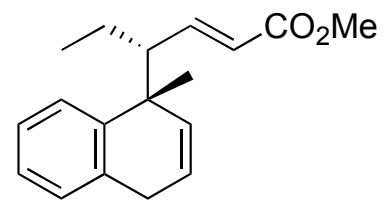

5

To a solution of 4-methyl-1,2-dihydronaphthalene $2(0.5 \mathrm{mmol})$ and $\mathrm{Rh}_{2}(S \text {-DOSP })_{4}(19$ $\mathrm{mg}, 0.01 \mathrm{mmol})$ in 2,2-dimethylbutane $(2 \mathrm{~mL})$ was added a solution of $(E)$-methyl 2diazo-3-hexnate $4(155 \mathrm{mg}, 1.0 \mathrm{mmol})$ in DMB $(5 \mathrm{~mL})$ at rt over a $2 \mathrm{~h}$ period via syringepump. The mixture was stirred for an additional $5 \mathrm{~min}$. The solvent was evaporated under resuced pressure and the residue was purified by flash chromatography on silica gel (20:1 to 10:1 pentane/ether eluent) to provide 5 (77 $\mathrm{mg}, 57 \%$ yield) as a colorless oil.

\section{$(R, 2 E)$-methyl 4-((S)-1,4-dihydro-1-methylnaphthalen-1-yl)hex-2-enoate (5):}

colorless oil; $\mathrm{R}_{\mathrm{f}} 0.31$ (10:1 pentane/ether); $[\alpha]_{\mathrm{D}}{ }^{25}-2.8$ (c 2.75, $\mathrm{CHCl}_{3}$ ); FTIR (film) 3028, 2964, 2874, 1724, 1654, 1493, 1436, 1350, 1268, 1243, 1174, $1138 \mathrm{~cm}^{-1} ;{ }^{1} \mathrm{H}$ NMR (500 $\left.\mathrm{MHz} \mathrm{CDCl}_{3}\right) \delta 7.29(\mathrm{~d}, J=7.9 \mathrm{~Hz}, 1 \mathrm{H}), 7.20(\mathrm{t}, J=7.0 \mathrm{~Hz}, 1 \mathrm{H}), 7.14(\mathrm{td}, J=7.3,1.2$ $\mathrm{Hz}, 1 \mathrm{H}), 7.10$ (d, $J=7.3 \mathrm{~Hz}, 1 \mathrm{H}), 6.84$ (dd, $J=15.6,10.4 \mathrm{~Hz}, 1 \mathrm{H}), 5.98$ (dt, $J=10.4,3.7$ $\mathrm{Hz}, 1 \mathrm{H}), 5.92$ (d, $J=15.6 \mathrm{~Hz}, 1 \mathrm{H}), 5.60$ (dt, $J=10.4,2.1 \mathrm{~Hz}, 1 \mathrm{H}), 3.76$ (s, 3H), 3.42-3.30 $(\mathrm{m}, 2 \mathrm{H}), 2.30(\mathrm{td}, J=10.7,3.1 \mathrm{~Hz}, 1 \mathrm{H}), 1.32(\mathrm{~s}, 3 \mathrm{H}), 1.22-1.05(\mathrm{~m}, 2 \mathrm{H}), 0.60(\mathrm{t}, J=7.3$ $\mathrm{Hz}, 3 \mathrm{H}) ;{ }^{13} \mathrm{C} \mathrm{NMR}\left(125 \mathrm{MHz}, \mathrm{CDCl}_{3}\right) \delta 166.7,150.0,141.5,134.4,130.9,128.1,126.3$, $125.8,125.7,124.6,123.3,58.0,51.4,41.9,30.1,29.4,22.3,12.4$; GC-MS (EI) $m / z$ (relative intensity): 128.0 (82), 143.1 (100); HPLC analysis: 98.4\% ee (Chiralcel OD-H, $1.0 \% i$-PrOH in hexane, $0.8 \mathrm{~mL} / \mathrm{min}, \lambda=254 \mathrm{~nm}, \mathrm{t}_{\mathrm{R}}=7.2 \mathrm{~min}$, major; $\mathrm{t}_{\mathrm{R}}=9.1 \mathrm{~min}$, minor); Anal. Calcd for $\mathrm{C}_{18} \mathrm{H}_{22} \mathrm{O}_{2}$ : C, 79.96; H, 8.20. Found: C, 79.93; H, 8.30.

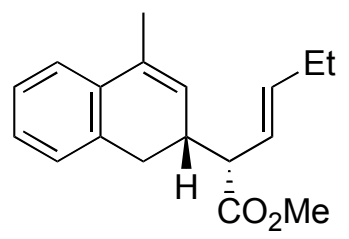


A solution of 5 ( $74 \mathrm{mg}, 98.4 \%$ ee) in toluene $(5 \mathrm{~mL})$ was refluxed for $6 \mathrm{~h}$. Toluene was removed by rotavapor and the residue was purified by flash chromatography on silica gel (20:1 pentane/ether eluent) to provide 6 (68 $\mathrm{mg}, 92 \%$ yield) as a colorless oil.

$(S, 3 E)$-methyl 2-((R)-1,2-dihydro-4-methylnaphthalen-2-yl)hex-3-enoate (6): colorless oil; $\mathrm{R}_{\mathrm{f}} 0.38$ (10:1 pentane/ether); $[\alpha]_{\mathrm{D}}{ }^{25}+141.1$ (c 3.40, $\mathrm{CHCl}_{3}$ ); FTIR (film) 3024, 2963, 2874, 1736, 1488, 1451, 1434, 1378, 1336, 1250, 1225, 1191, 1157, 1026, $971 \mathrm{~cm}^{-1} ;{ }^{1} \mathrm{H}$ NMR $\left(500 \mathrm{MHz}, \mathrm{CDCl}_{3}\right) \delta$ 7.25-7.18 (m, 2H), $7.14(\mathrm{td}, J=7.0,1.5 \mathrm{~Hz}$, $1 \mathrm{H}), 7.08$ (br d, $J=7.3 \mathrm{~Hz}, 1 \mathrm{H}), 5.69-5.66(\mathrm{~m}, 1 \mathrm{H}), 5.55(\mathrm{dt}, J=15.3,6.2 \mathrm{~Hz}, 1 \mathrm{H}), 5.36$ (ddt, $J=15.3,9.5,1.5 \mathrm{~Hz}, 1 \mathrm{H}), 3.67(\mathrm{~s}, 3 \mathrm{H}), 2.89$ (t, $J=9.5 \mathrm{~Hz}, 1 \mathrm{H}), 2.84-2.78(\mathrm{~m}, 1 \mathrm{H}$ ), 2.72-2.63 (m, 2H), 2.08-2.01 (m, 2H), $2.04(\mathrm{~s}, 3 \mathrm{H}), 0.99(\mathrm{t}, J=7.3 \mathrm{~Hz}, 3 \mathrm{H}) ;{ }^{13} \mathrm{C} \mathrm{NMR}$ (125 MHz, $\left.\mathrm{CDCl}_{3}\right) \delta 174.2(\mathrm{C}), 136.7(\mathrm{CH}), 135.3(\mathrm{C}), 134.5(\mathrm{C}), 133.2(\mathrm{C}), 127.7(\mathrm{CH})$, 127.0 (CH), $126.6(\mathrm{CH}), 126.4(\mathrm{CH}), 125.0(\mathrm{CH}), 122.9(\mathrm{CH}), 52.6(\mathrm{CH}), 51.6\left(\mathrm{CH}_{3}\right)$, $35.8(\mathrm{CH}), 31.5\left(\mathrm{CH}_{2}\right), 25.5\left(\mathrm{CH}_{2}\right), 19.3\left(\mathrm{CH}_{3}\right), 13.5\left(\mathrm{CH}_{3}\right)$; LC-MS (ESI) $\mathrm{m} / z$ (relative intensity): $271.1\left([\mathrm{M}+\mathrm{H}]^{+}, 100\right)$; HPLC analysis: $98.0 \%$ ee (Chiralcel OD-H, $1.0 \% i$ PrOH in hexane, $0.8 \mathrm{~mL} / \mathrm{min}, \lambda=254 \mathrm{~nm}, t_{\mathrm{R}}=7.5 \mathrm{~min}$, minor; $\mathrm{t}_{\mathrm{R}}=9.4 \mathrm{~min}$, major); Anal. Calcd for $\mathrm{C}_{18} \mathrm{H}_{22} \mathrm{O}_{2}:$ C, 79.96; H, 8.20. Found: C, 79.81; H, 8.24.

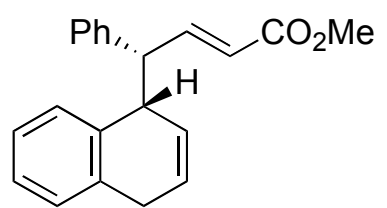

8

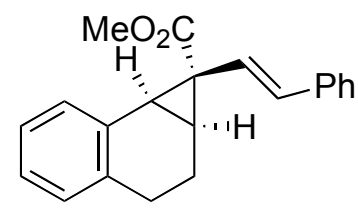

9

To a solution of 1,2-dihydronaphthalene $7(0.5 \mathrm{mmol})$ and $\mathrm{Rh}_{2}(S \text {-DOSP })_{4}(4.8 \mathrm{mg}$, $0.0025 \mathrm{mmol})$ in 2,2-dimethylbutane $(1 \mathrm{~mL})$ was added a solution of $(E)$-methyl phenylvinyldiazoacetate $1(111 \mathrm{mg}, 0.55 \mathrm{mmol})$ in DMB $(5 \mathrm{~mL})$ over a $45 \mathrm{~min}$ period via syringe-pump at $0{ }^{\circ} \mathrm{C}$ (with crashed-ice cooling). The resulting mixture was stirred for an additional $5 \mathrm{~min}$. The solvent was then evaporated under reduced pressure and the residue (8:9 = 5.5:1 based on crude ${ }^{1} \mathrm{H}$ NMR) was purified by flash chromatography on silica gel (20:1 to 10:1 pentane/ether eluent) to provide a mixture of 8 and $\mathbf{9}$ (121 $\mathrm{mg}, 79 \%$ yield).

$(R, 2 E)$-methyl 4-((S)-1,4-dihydronaphthalen-4-yl)-4-phenylbut-2-enoate (8): 
$\mathrm{R}_{\mathrm{f}} 0.35$ (5:1 pentane/ether); ${ }^{1} \mathrm{H}$ NMR $\left(500 \mathrm{MHz}, \mathrm{CDCl}_{3}\right) \delta 7.30(\mathrm{dd}, J=15.8,8.5 \mathrm{~Hz}$, 1H), 7.18-7.11 (m, 5H), 7.07-7.04 (m, 1H), 6.97 (br d, $J=7.6 \mathrm{~Hz}, 1 \mathrm{H}), 6.94-6.90$ (m, $2 \mathrm{H}), 6.00(\mathrm{ddd}, J=9.8,5.2,1.8 \mathrm{~Hz}, 1 \mathrm{H}), 5.90(\mathrm{ddd}, J=9.8,4.9,3.0 \mathrm{~Hz}, 1 \mathrm{H}), 5.82(\mathrm{dd}, J$ $=15.8,0.6 \mathrm{~Hz}, 1 \mathrm{H}), 3.92-3.88(\mathrm{~m}, 1 \mathrm{H}), 3.75-3.70(\mathrm{~m}, 1 \mathrm{H}), 3.72(\mathrm{~s}, 3 \mathrm{H}), 3.03(\mathrm{ddd}, J=$ 21.0, 5.2, $2.1 \mathrm{~Hz}, 1 \mathrm{H}), 2.67-2.58(\mathrm{~m}, 1 \mathrm{H}) ;{ }^{13} \mathrm{C} \mathrm{NMR}\left(125 \mathrm{MHz}, \mathrm{CDCl}_{3}\right) \delta 166.8(\mathrm{C})$, $149.4(\mathrm{CH}), 139.0(\mathrm{C}), 136.0(\mathrm{C}), 135.8(\mathrm{C}), 128.8(\mathrm{CH}), 128.6(\mathrm{CH}), 128.4(\mathrm{CH}), 127.9$ $(\mathrm{CH}), 127.8(\mathrm{CH}), 126.6(\mathrm{CH}), 126.1(\mathrm{CH}), 125.6(\mathrm{CH}), 121.9(\mathrm{CH}), 55.8(\mathrm{CH}), 51.4$ $\left(\mathrm{CH}_{3}\right), 46.2(\mathrm{CH}), 29.9\left(\mathrm{CH}_{2}\right)$. Molecular analysis is not available due to its slow rearrangement to $\mathbf{1 0}$.

(1R, $1 \alpha S, 7 \alpha S)$-methyl $1 \alpha, 6,7,7 \alpha$-tetrahydro-1-styryl-1H-cyclopropa[ $\alpha]$ naththalene1-carboxylate (9):

colorless oil; $\mathrm{R}_{\mathrm{f}} 0.35$ (5:1 pentane/ether); FTIR (film) 3024, 2926, 2846, 1718, 1494, $1433,1236 \mathrm{~cm}^{-1} ;{ }^{1} \mathrm{H}$ NMR $\left(500 \mathrm{MHz}, \mathrm{CDCl}_{3}\right) \delta 7.38(\mathrm{br} \mathrm{d}, J=7.3 \mathrm{~Hz}, 1 \mathrm{H}), 7.23-7.11$ (m, 5H), 7.09 (d, $J=7.3 \mathrm{~Hz}, 2 \mathrm{H}), 6.96$ (br d, $J=7.3 \mathrm{~Hz}, 1 \mathrm{H}), 6.17$ (d, $J=16.2 \mathrm{~Hz}, 1 \mathrm{H}$ ), 5.97 (d, $J=16.2 \mathrm{~Hz}, 1 \mathrm{H}), 3.72$ (s, 3H), 2.94 (d, $J=9.4 \mathrm{~Hz}, 1 \mathrm{H}$ ), 2.61 (ddd, $J=16.5,7.0$, $4.0 \mathrm{~Hz}, 1 \mathrm{H}), 2.46(\mathrm{~m}, 2 \mathrm{H}), 2.13-2.02(\mathrm{~m}, 2 \mathrm{H}) ;{ }^{13} \mathrm{C} \mathrm{NMR}\left(125 \mathrm{MHz}, \mathrm{CDCl}_{3}\right) \delta 173.8$, 137.3, 135.6, 135.5, 132.7, 130.2, 128.6, 128.4, 127.4, 126.5, 126.03, 126.01, 120.8, 52.4, 35.6, 30.3, 28.6, 26.6, 17.9; LRMS (EI) $\mathrm{m} / z$ (relative intensity): 115.0 (74), 128.1 (53), 129.1 (59), 245.1 (100), $304.2\left(\mathrm{M}^{+}, 74\right)$; HRMS (EI) $\mathrm{m} / z$ Calcd for $\left[\mathrm{C}_{21} \mathrm{H}_{20} \mathrm{O}_{2}\right]^{+}\left(\mathrm{M}^{+}\right)$: 304.1458. Found: 304.1464.

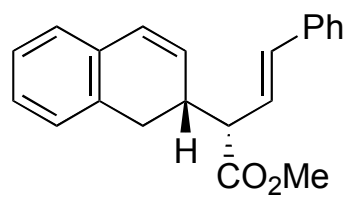

10

A solution of 8 and $9(6: 1)$ in toluene was heated at $60{ }^{\circ} \mathrm{C}$ for $48 \mathrm{~h}$. Toluene was removed under reduced pressure and the residue was purified by flash chromatography on silica gel (20:1 to 10:1 pentane/ether eluent) to provide $\mathbf{1 0}$ (92 $\mathrm{mg}, 60 \%$ overall yield) and 9 (14 $\mathrm{mg}, 9 \%$ yield).

$(S, 3 E)$-methyl 2-((R)-1,2-dihydronaphthalen-2-yl)-4-phenylbut-3-enoate (10): 
white solid, $\mathrm{mp} 83-84{ }^{\circ} \mathrm{C} ; \mathrm{R}_{\mathrm{f}} 0.23\left(10: 1\right.$ pentane/ether); $[\alpha]_{\mathrm{D}}{ }^{25}+9.5\left(\right.$ c $\left.3.00, \mathrm{CHCl}_{3}\right)$; FTIR $\left(\mathrm{CH}_{2} \mathrm{Cl}_{2}\right) 3028,2949,1734,1451,1434,1258,1193,1162 \mathrm{~cm}^{-1} ;{ }^{1} \mathrm{H}$ NMR $(500$ $\left.\mathrm{MHz}, \mathrm{CDCl}_{3}\right) \delta$ 7.34-7.28 (m, 4H), 7.26-7.22 (m, 1H), 7.18-7.11 (m, 2H), 7.07-7.02 (m, 2H), $6.52(\mathrm{~d}, J=9.8 \mathrm{~Hz}, 1 \mathrm{H}), 6.42(\mathrm{~d}, J=15.9 \mathrm{~Hz}, 1 \mathrm{H}), 6.14(\mathrm{dd}, J=15.9,9.8 \mathrm{~Hz}, 1 \mathrm{H})$, 5.92 (dd, $J=9.8,4.3 \mathrm{~Hz}, 1 \mathrm{H}), 3.69$ (s, 3H), 3.14 (appt. t, $J=9.5 \mathrm{~Hz}, 1 \mathrm{H}), 2.94-2.86$ (m, 2H), 2.82-2.74 (m, 1H); ${ }^{13} \mathrm{C}$ NMR $\left(125 \mathrm{MHz}, \mathrm{CDCl}_{3}\right) \delta 173.5,136.5,133.8,133.4$, 129.4, 128.8, 128.5, 127.9, 127.7, 127.4, 126.6, 126.4, 126.1, 125.7, 52.9, 51.8, 36.0, 30.8; LC-MS (ESI) m/z (relative intensity): $305.0\left([\mathrm{M}+\mathrm{H}]^{+}, 80\right), 322.0\left([\mathrm{M}+\mathrm{Na}]^{+}, 100\right)$; HPLC analysis: $99.3 \%$ ee (Chiralcel OD-H, $2.0 \% i-\mathrm{PrOH}$ in hexane, $0.8 \mathrm{~mL} / \mathrm{min}, \lambda=254$ $\mathrm{nm}, \mathrm{t}_{\mathrm{R}}=9.7$ min, minor; $\mathrm{t}_{\mathrm{R}}=11.6$ min, major); Anal. Calcd for $\mathrm{C}_{21} \mathrm{H}_{20} \mathrm{O}_{2}: \mathrm{C}, 82.86 ; \mathrm{H}$, 6.62. Found: C, 82.64; H, 6.55 .

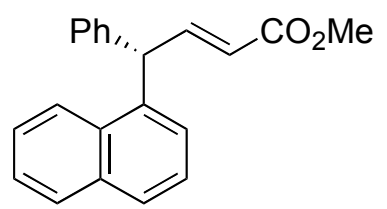

13

To a solution of 3,4-dihydronaphthalen-1-yl acetate $11(0.5 \mathrm{mmol})$ and $\mathrm{Rh}_{2}(S \text {-DOSP })_{4}$ (4.8 $\mathrm{mg}, 0.0025 \mathrm{mmol})$ in 2,2-dimethylbutane $(2 \mathrm{~mL})$ and $\mathrm{PhCF}_{3}(0.4 \mathrm{~mL})$ was added a solution of $(E)$-methyl phenylvinyldiazoacetate $1(111 \mathrm{mg}, 0.55 \mathrm{mmol})$ in DMB $(5 \mathrm{~mL})$ over a 45 min period via syringe-pump at $0{ }^{\circ} \mathrm{C}$ (with crashed-ice cooling). The resulting mixture was stirred for an additional $5 \mathrm{~min}$. The solvent was then evaporated under reduced pressure and the residue was purified by flash chromatography on silica gel (10:1 to 5:1 pentane/ether eluent) to provide $\mathbf{1 3}$ ( $129 \mathrm{mg}, 85 \%$ yield).

$(S, E)$-methyl 4-(naphthalen-4-yl)-4-phenylbut-2-enoate (13):

colorless oil; $\mathrm{R}_{\mathrm{f}} 0.35$ (5:1 pentane/ether); $[\alpha]_{\mathrm{D}}^{25}+70.5$ (c 5.00, $\mathrm{CHCl}_{3}$ ); FTIR (film) 3060, 3024, 2950, 1722, 1652, 1600, 1511, 1495, 1453, 1432, 1132, 1270, $1170 \mathrm{~cm}^{-1} ;{ }^{1} \mathrm{H}$ NMR $\left(500 \mathrm{MHz}, \mathrm{CDCl}_{3}\right) \delta$ 7.95-7.90 (m, 1H), 7.86-7.81 (m, 1H), $7.77(\mathrm{~d}, J=8.2 \mathrm{~Hz}, 1 \mathrm{H}), 7.56$ (dd, $J=15.6,6.4 \mathrm{~Hz}, 1 \mathrm{H}), 7.45-7.39(\mathrm{~m}, 3 \mathrm{H}), 7.30-7.25(\mathrm{~m}, 3 \mathrm{H}), 7.23-7.19(\mathrm{~m}, 1 \mathrm{H}), 7.17$ (br d, $J=7.6 \mathrm{~Hz}, 2 \mathrm{H}), 5.64(\mathrm{~d}, J=15.6 \mathrm{~Hz}, 1 \mathrm{H}), 5.62(\mathrm{~d}, J=6.4 \mathrm{~Hz}, 1 \mathrm{H}), 3.71(\mathrm{~s}, 3 \mathrm{H})$; ${ }^{13} \mathrm{C}$ NMR $\left(125 \mathrm{MHz}, \mathrm{CDCl}_{3}\right) \delta$ 166.8, 150.4, 140.9, 137.0, 134.0, 131.4, 128.83, 128.76, 
128.67, 127.9, 126.9, 126.5, 126.2, 125.6, 125.3, 123.7, 123.0, 51.5, 49.4; LC-MS (ESI) $\mathrm{m} / z$ (relative intensity): $271.4(21), 303.2\left([\mathrm{M}+\mathrm{H}]^{+}, 100\right), 335.1\left([\mathrm{M}+\mathrm{Na}]^{+}, 79\right)$; HRMS (ESI) $\mathrm{m} / z$ Calcd for $\left[\mathrm{C}_{21} \mathrm{H}_{18} \mathrm{NaO}_{2}\right]^{+}\left([\mathrm{M}+\mathrm{Na}]^{+}\right): 325.1199$. Found: 325.1210. HPLC analysis: $99.6 \%$ ee (Chiralcel OD-H, $2.0 \% i$-PrOH in hexane, $0.8 \mathrm{~mL} / \mathrm{min}, \lambda=254 \mathrm{~nm}, \mathrm{t}_{\mathrm{R}}$ $=13.0 \mathrm{~min}$, major; $\mathrm{t}_{\mathrm{R}}=39.4 \mathrm{~min}$, minor).

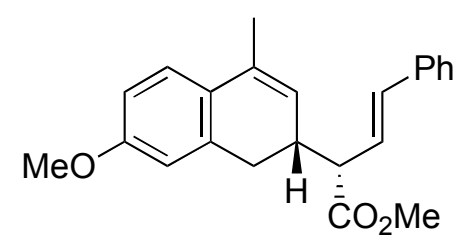

$15 \mathbf{a}$

To a solution of 7-methoxy-4-methyl-1,2-dihydronaphthalene $14 \mathbf{a}(0.5 \mathrm{mmol})$ and $\mathrm{Rh}_{2}(\mathrm{~S}$ DOSP $)_{4}(4.8 \mathrm{mg}, 0.0025 \mathrm{mmol})$ in 2,2-dimethylbutane $(1 \mathrm{~mL})$ was added a solution of (E)-methyl phenylvinyldiazoacetate $1(111 \mathrm{mg}, 0.55 \mathrm{mmol})$ in DMB $(5 \mathrm{~mL})$ over a 45 min period via syringe-pump at $0{ }^{\circ} \mathrm{C}$ (with crashed-ice cooling). The resulting mixture was then stirred overnight at $\mathrm{rt}(\mathrm{ca} 12 \mathrm{~h})$. The solvent was then evaporated under reduced pressure and the residue was purified by flash chromatography on silica gel (5:1 pentane/ether eluent) to provide $15 \mathrm{a}$ (157 $\mathrm{mg}, 90 \%$ yield).

$(S, 3 E)$-methyl 2-((R)-1,2-dihydro-7-methoxy-4-methylnaphthalen-2-yl)-4-phenylbut3-enoate (15a):

white solid, $\mathrm{mp} 87-88{ }^{\circ} \mathrm{C} ; \mathrm{R}_{\mathrm{f}} 0.30$ (5:1 pentane/ether); $[\alpha]_{\mathrm{D}}{ }^{25}-15.1$ (c $6.60, \mathrm{CHCl}_{3}$ ); FTIR $\left(\mathrm{CDCl}_{3}\right)$ : 3027, 2998, 2949, 2836, 1733, 1607, 1570, 1499, 1449, 1433, 1309, 1251, 1191, 1159, 1143, 1071, $1032 \mathrm{~cm}^{-1} ;{ }^{1} \mathrm{H}$ NMR $\left(500 \mathrm{MHz}, \mathrm{CDCl}_{3}\right) \delta$ 7.37-7.29 (m, 4H), 7.27-7.22 (m, 1H), $7.16(\mathrm{~d}, J=8.5 \mathrm{~Hz}, 1 \mathrm{H}), 6.73(\mathrm{dd}, J=8.5,2.4 \mathrm{~Hz}, 1 \mathrm{H}), 6.65(\mathrm{~d}, J$ $=2.4 \mathrm{~Hz}, 1 \mathrm{H}), 6.43(\mathrm{~d}, J=15.9 \mathrm{~Hz}, 1 \mathrm{H}), 6.14(\mathrm{dd}, J=15.9,9.8 \mathrm{~Hz}, 1 \mathrm{H}), 5.59(\mathrm{br} \mathrm{d}, J=$ $2.7 \mathrm{~Hz}, 1 \mathrm{H}$ ), 3.79 (s, 3H), 3.70 (s, 3H), 3.10 (appt. t, $J=9.5 \mathrm{~Hz}, 1 \mathrm{H}), 2.88-2.78$ (m, 2H), $2.68(\mathrm{dd}, J=14.4,6.4 \mathrm{~Hz}, 1 \mathrm{H}), 2.03(\mathrm{~s}, 3 \mathrm{H}) ;{ }^{13} \mathrm{C} \mathrm{NMR}\left(125 \mathrm{MHz}, \mathrm{CDCl}_{3}\right) \delta 173.6$, 158.7, 136.6, 136.2, 133.6, 133.2, 128.5, 128.4, 127.6, 126.3, 126.0, 124.1, 123.7, 113.9, 111.0, 55.1, 53.2, 51.8, 36.1, 32.1, 19.4; LC-MS (ESI) $\mathrm{m} / \mathrm{z}$ (relative intensity): 349.1 $\left([\mathrm{M}+\mathrm{H}]^{+}, 100\right)$; HPLC analysis: $98.9 \%$ ee (Chiralcel OD-H, $2.0 \% i-\mathrm{PrOH}$ in hexane, 0.8 
$\mathrm{mL} / \mathrm{min}, \lambda=254 \mathrm{~nm}, \mathrm{t}_{\mathrm{R}}=10.4 \mathrm{~min}$, major; $\mathrm{t}_{\mathrm{R}}=14.9 \mathrm{~min}$, minor); Anal. Calcd for $\mathrm{C}_{23} \mathrm{H}_{24} \mathrm{O}_{3}$ : C, 79.28; H, 6.94. Found: C, 79.09; H, 7.02.

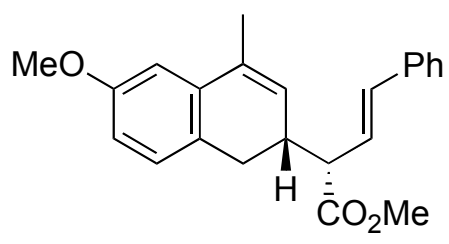

15b

To a solution of 6-methoxy-4-methyl-3,4-dihydronaphthalene $14 \mathbf{b}(0.5 \mathrm{mmol})$ and $\mathrm{Rh}_{2}(S$ DOSP $)_{4}(4.8 \mathrm{mg}, 0.0025 \mathrm{mmol})$ in 2,2-dimethylbutane $(1 \mathrm{~mL})$ was added a solution of (E)-methyl phenylvinyldiazoacetate $1(111 \mathrm{mg}, 0.55 \mathrm{mmol})$ in DMB $(5 \mathrm{~mL})$ over a 45 min period via syringe-pump at $0{ }^{\circ} \mathrm{C}$ (with crashed-ice cooling). The resulting mixture was then stirred overnight at $\mathrm{rt}(c a .12 \mathrm{~h})$. The solvent was then evaporated under reduced pressure and the residue was purified by flash chromatography on silica gel $(5: 1$ pentane/ether eluent) to provide $\mathbf{1 5 b}$ (134 $\mathrm{mg}, 77 \%$ yield).

$(S, 3 E)$-methyl 2-((R)-1,2-dihydro-6-methoxy-4-methylnaphthalen-2-yl)-4-phenylbut3-enoate (15b):

colorless oil; $\mathrm{R}_{\mathrm{f}} 0.30$ (5:1 pentane/ether); $[\alpha]_{\mathrm{D}}{ }^{25}-54.3$ (c 3.80, $\left.\mathrm{CHCl}_{3}\right)$; FTIR (film): $3025,2949,2835,1733,1605,1569,1490,1449,1433,1256,1205,1158 \mathrm{~cm}^{-1} ;{ }^{1} \mathrm{H}$ NMR $\left(500 \mathrm{MHz}, \mathrm{CDCl}_{3}\right) \delta$ 7.36-7.28 (m, 4H), 7.26-7.22 (m, 1H), $6.99(\mathrm{~d}, J=8.2 \mathrm{~Hz}, 1 \mathrm{H}), 6.81$ $(\mathrm{d}, J=2.4 \mathrm{~Hz}, 1 \mathrm{H}), 6.70(\mathrm{dd}, J=8.2,2.4 \mathrm{~Hz}, 1 \mathrm{H}), 6.41(\mathrm{~d}, J=15.9 \mathrm{~Hz}, 1 \mathrm{H}), 6.14(\mathrm{dd}, J$ $=15.9,9.6 \mathrm{~Hz}, 1 \mathrm{H}), 5.75(\mathrm{br} \mathrm{d}, J=3.1 \mathrm{~Hz}, 1 \mathrm{H}), 3.80(\mathrm{~s}, 3 \mathrm{H}), 3.69$ (s, 3H), 3.10 (appt. t, $J$ $=9.2 \mathrm{~Hz}, 1 \mathrm{H}), 2.84-2.76(\mathrm{~m}, 2 \mathrm{H}), 2.64(\mathrm{dd}, J=17.1,9.8 \mathrm{~Hz}, 1 \mathrm{H}), 2.05(\mathrm{~s}, 3 \mathrm{H}) ;{ }^{13} \mathrm{C} \mathrm{NMR}$ $\left(125 \mathrm{MHz}, \mathrm{CDCl}_{3}\right) \delta 173.6,158.4,136.6,136.3,133.6,133.5,128.5,128.4,127.7,126.9$, $126.5,126.4,126.0,111.4,109.8,55.3,53.1,51.8,36.3,30.7,19.4$; LRMS (ESI) $\mathrm{m} / z$ (relative intensity): $349.3\left([\mathrm{M}+\mathrm{H}]^{+}, 36\right)$; HRMS (ESI) $\mathrm{m} / z$ Calcd for $\left[\mathrm{C}_{23} \mathrm{H}_{24} \mathrm{NaO}_{3}\right]^{+}$ $\left([\mathrm{M}+\mathrm{Na}]^{+}\right)$: 371.1618. Found: 376.1614. HPLC analysis: 99.3\% ee (Chiralcel OD-H, $2.0 \% i-\mathrm{PrOH}$ in hexane, $0.8 \mathrm{~mL} / \mathrm{min}, \lambda=254 \mathrm{~nm}, \mathrm{t}_{\mathrm{R}}=11.4 \mathrm{~min}$, major; $\mathrm{t}_{\mathrm{R}}=15.9 \mathrm{~min}$, minor). 


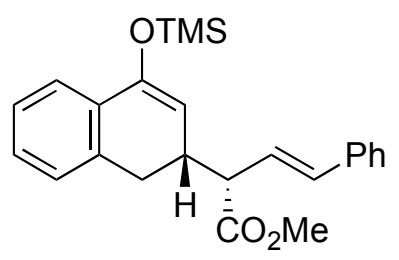

$17 \mathbf{a}$

To a solution of (3,4-dihydronaphthalen-1-yloxy)-trimehtylsilane $\mathbf{1 6 a}(0.5 \mathrm{mmol})$ and $\mathrm{Rh}_{2}(S \text {-DOSP })_{4}(4.8 \mathrm{mg}, 0.0025 \mathrm{mmol})$ in 2,2-dimethylbutane $(1 \mathrm{~mL})$ was added a solution of $(E)$-methyl phenylvinyldiazoacetate $1(111 \mathrm{mg}, 0.55 \mathrm{mmol})$ in DMB $(5 \mathrm{~mL})$ over a 45 min period via syringe-pump at $0{ }^{\circ} \mathrm{C}$ (with crashed-ice cooling). The resulting mixture was then stirred for another $2 \mathrm{~h}$ at $\mathrm{rt}$. The solvent was then evaporated under reduced pressure and the residue was purified by flash chromatography on silica gel (20:1 pentane/ether eluent) to provide $\mathbf{1 7 a}$ (108 $\mathrm{mg}, 55 \%$ yield).

$(S, 3 E)$-methyl 2-((R)-1,2-dihydro-4-trimethylsiloxylnaphthalen-2-yl)-4-phenylbut-3enoate (17a):

colorless oil; $\mathrm{R}_{\mathrm{f}} 0.30$ (10:1 pentane/ether); $[\alpha]_{\mathrm{D}}{ }^{25}-46.5$ (c 3.00, $\mathrm{CHCl}_{3}$ ); FTIR (film): 3061, 3027, 2952, 1734, 1636, 1488, 1450, 1435, 1363, 1341, 1254, 1159, 1089, 1064 $\mathrm{cm}^{-1} ;{ }^{1} \mathrm{H}$ NMR $\left(500 \mathrm{MHz}, \mathrm{CDCl}_{3}\right) \delta 7.42(\mathrm{~d}, J=7.5 \mathrm{~Hz}, 1 \mathrm{H}), 7.35-7.28(\mathrm{~m}, 4 \mathrm{H}), 7.26-$ $7.19(\mathrm{~m}, 2 \mathrm{H}), 7.17(\mathrm{td}, J=7.3,1.2 \mathrm{~Hz}, 1 \mathrm{H}), 7.06(\mathrm{~d}, J=7.3 \mathrm{~Hz}, 1 \mathrm{H}), 6.39$ (d, $J=15.6$ $\mathrm{Hz}, 1 \mathrm{H}), 6.13(\mathrm{dd}, J=15.6,9.7 \mathrm{~Hz}, 1 \mathrm{H}), 5.14(\mathrm{~d}, J=5.1 \mathrm{~Hz}, 1 \mathrm{H}), 3.66(\mathrm{~s}, 3 \mathrm{H}), 3.10$ (appt. t, $J=9.5 \mathrm{~Hz}, 1 \mathrm{H}), 2.95-2.85(\mathrm{~m}, 2 \mathrm{H}), 2.77$ (d, $J=14.7,4.3 \mathrm{~Hz}, 1 \mathrm{H}), 0.26(\mathrm{~s}, 9 \mathrm{H})$; ${ }^{13} \mathrm{C}$ NMR $\left(125 \mathrm{MHz}, \mathrm{CDCl}_{3}\right) \delta 137.7,149.0,136.6,134.9,133.8,132.8,128.5,127.8$, $127.7,127.5,126.4,126.3,126.1,122.1,106.2,53.3,51.8,35.5,31.0,0.1$; LRMS (ESI) $m / z$ (relative intensity): 343.4 (100), $415.3\left([\mathrm{M}+\mathrm{Na}]^{+}, 44\right)$; HRMS (ESI) $m / z$ Calcd for $\left[\mathrm{C}_{24} \mathrm{H}_{28} \mathrm{NaO}_{3} \mathrm{Si}\right]^{+}\left([\mathrm{M}+\mathrm{Na}]^{+}\right): 415.1700$. Found: 415.1716. HPLC analysis: $97.5 \%$ ee (Chiralcel OD-H, 2.0\% $i$-PrOH in hexane, $0.8 \mathrm{~mL} / \mathrm{min}, \lambda=254 \mathrm{~nm}, \mathrm{t}_{\mathrm{R}}=7.3 \mathrm{~min}$, major; $t_{R}=11.5$ min, minor). 


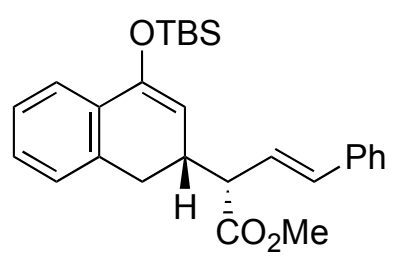

17b

To a solution of tert-butyl-(3,4-dihydronaphthalen-1-yloxy)-dimethylsilane $\mathbf{1 6} \mathbf{b}$ (0.5 $\mathrm{mmol})$ and $\mathrm{Rh}_{2}(S \text {-DOSP })_{4}(4.8 \mathrm{mg}, 0.0025 \mathrm{mmol})$ in 2,2-dimethylbutane $(1 \mathrm{~mL})$ was added a solution of $(E)$-methyl phenylvinyldiazoacetate $1(111 \mathrm{mg}, 0.55 \mathrm{mmol})$ in DMB $\left(5 \mathrm{~mL}\right.$ ) over a $45 \mathrm{~min}$ period via syringe-pump at $0{ }^{\circ} \mathrm{C}$ (with crashed-ice cooling). The resulting mixture was then stirred for another $2 \mathrm{~h}$ at $\mathrm{rt}$. The solvent was then evaporated under reduced pressure and the residue was purified by flash chromatography on silica gel (20:1 pentane/ether eluent) to provide $\mathbf{1 7 b}$ (171 $\mathrm{mg}, 78 \%$ yield).

$(S, 3 E)$-methyl 2-((R)-1,2-dihydro-4-tert-butyldimethylsiloxylnaphthalen-2-yl)-4phenylbut-3-enoate (17b):

colorless oil; $\mathrm{R}_{\mathrm{f}} 0.33$ (10:1 pentane/ether); $[\alpha]_{\mathrm{D}}{ }^{25}-51.7$ (c 4.00, $\left.\mathrm{CHCl}_{3}\right)$; FTIR (film): 3061, 3026, 2953, 2930, 2885, 2858, 1735, 1636, 1601, 1572, 1488, 1472, 1450, 1434, $1363,1340,1259,1158,1088,1064 \mathrm{~cm}^{-1} ;{ }^{1} \mathrm{H}$ NMR $\left(500 \mathrm{MHz}, \mathrm{CDCl}_{3}\right) \delta 7.48(\mathrm{br} \mathrm{d}, J=$ 7.6 Hz, 1H), 7.35-7.28 (m, 4H), 7.25-7.18 (m, 2H), $7.16(\mathrm{td}, J=7.3,1.2 \mathrm{~Hz}, 1 \mathrm{H}), 7.05(\mathrm{~d}$, $J=7.3 \mathrm{~Hz}, 1 \mathrm{H}), 6.39(\mathrm{~d}, J=15.6 \mathrm{~Hz}, 1 \mathrm{H}), 6.13(\mathrm{dd}, J=15.6,9.6 \mathrm{~Hz}, 1 \mathrm{H}), 5.12(\mathrm{~d}, J=$ $4.9 \mathrm{~Hz}, 1 \mathrm{H}$ ), 3.66 (s, 3H), 3.10 (appt. t, $J=9.5 \mathrm{~Hz}, 1 \mathrm{H}), 2.93-2.84(\mathrm{~m}, 2 \mathrm{H}), 2.79-2.72$ (m, 1H), 1.01 (s, 9H), $0.21(\mathrm{~s}, 3 \mathrm{H}), 0.20(\mathrm{~s}, 3 \mathrm{H}) ;{ }^{13} \mathrm{C} \mathrm{NMR}\left(125 \mathrm{MHz}, \mathrm{CDCl}_{3}\right) \delta 173.7(\mathrm{C})$, $149.2(\mathrm{C}), 136.6(\mathrm{C}), 134.9(\mathrm{C}), 133.8(\mathrm{CH}), 133.0(\mathrm{C}), 128.5(\mathrm{CH}), 127.8(\mathrm{CH}), 127.7$ $(\mathrm{CH}), 127.5(\mathrm{CH}), 126.4(\mathrm{CH}), 126.3(\mathrm{CH}), 126.1(\mathrm{CH}), 122.1(\mathrm{CH}), 105.7(\mathrm{CH}), 53.4$ $(\mathrm{CH}), 51.8\left(\mathrm{CH}_{3}\right), 35.6(\mathrm{CH}), 31.0\left(\mathrm{CH}_{2}\right), 25.8\left(\mathrm{CH}_{3}\right), 18.3(\mathrm{C}),-4.4\left(\mathrm{CH}_{3}\right),-4.8\left(\mathrm{CH}_{3}\right)$; LRMS (ESI) $m / z$ (relative intensity): $435.5\left([\mathrm{M}+\mathrm{H}]^{+}, 15\right), 457.3\left([\mathrm{M}+\mathrm{Na}]^{+}, 100\right)$; HRMS (ESI) $m / z$ Calcd for $\left[\mathrm{C}_{27} \mathrm{H}_{34} \mathrm{NaO}_{3} \mathrm{Si}\right]^{+}\left([\mathrm{M}+\mathrm{Na}]^{+}\right): 457.2169$. Found: 457.2185. HPLC analysis: $95.2 \%$ ee (Chiralcel OD-H, $2.0 \% i-\mathrm{PrOH}$ in hexane, $0.8 \mathrm{~mL} / \mathrm{min}, \lambda=254 \mathrm{~nm}, \mathrm{t}_{\mathrm{R}}$ $=6.2 \mathrm{~min}$, major; $\mathrm{t}_{\mathrm{R}}=8.8 \mathrm{~min}$, minor). 


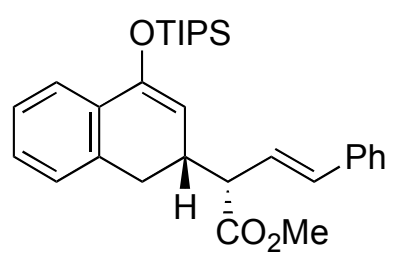

$17 \mathrm{c}$

To a solution of 1-(3,4-dihydronaphthalenyloxy)-triisopropylsilane $\mathbf{1 6 c}(0.5 \mathrm{mmol})$ and $\mathrm{Rh}_{2}(S \text {-DOSP })_{4}(4.8 \mathrm{mg}, 0.0025 \mathrm{mmol})$ in 2,2-dimethylbutane $(1 \mathrm{~mL})$ was added a solution of $(E)$-methyl phenylvinyldiazoacetate $1(111 \mathrm{mg}, 0.55 \mathrm{mmol})$ in DMB $(5 \mathrm{~mL})$ over a $45 \mathrm{~min}$ period via syringe-pump at $0{ }^{\circ} \mathrm{C}$ (with crashed-ice cooling). The resulting mixture was then stirred for another $2 \mathrm{~h}$ at $\mathrm{rt}$. The solvent was then evaporated under reduced pressure and the residue was purified by flash chromatography on silica gel (20:1 pentane/ether eluent) to provide $17 \mathrm{c}$ (127 $\mathrm{mg}, 53 \%$ yield).

$(S, 3 E)$-methyl 2-((R)-1,2-dihydro-4-triisopropylsiloxylnaphthalen-2-yl)-4-phenylbut3-enoate (17c):

colorless oil; $\mathrm{R}_{\mathrm{f}} 0.38$ (10:1 pentane/ether); $[\alpha]_{\mathrm{D}}{ }^{25}-51.9$ (c 4.60, $\left.\mathrm{CHCl}_{3}\right)$; FTIR (film): 3061, 3026, 2946, 2892, 2867, 1735, 1636, 1600, 1574, 1487, 1464, 1434, 1362, 1340, 1260, 1158, 1089, $1066 \mathrm{~cm}^{-1}$; ${ }^{1} \mathrm{H}$ NMR $\left(500 \mathrm{MHz}, \mathrm{CDCl}_{3}\right) \delta 7.56(\mathrm{~d}, J=7.3 \mathrm{~Hz}, 1 \mathrm{H})$, 7.36-7.28 (m, 4H), 7.25-7.19 (m, 2H), 7.17 (td, $J=7.3,1.2 \mathrm{~Hz}, 1 \mathrm{H}), 7.05$ (d, $J=7.3 \mathrm{~Hz}$, $1 \mathrm{H}), 6.40(\mathrm{~d}, J=15.9 \mathrm{~Hz}, 1 \mathrm{H}), 6.14$ (dd, $J=15.9,9.8 \mathrm{~Hz}, 1 \mathrm{H}), 5.11$ (d, $J=4.9 \mathrm{~Hz}, 1 \mathrm{H})$, 3.65 (s, 3H), 3.11 (appt. t, $J=9.8 \mathrm{~Hz}, 1 \mathrm{H}$ ), 2.93-2.86 (m, 2H), 2.75 (dd, $J=17.5,8.0 \mathrm{~Hz}$, 1H), 1.28 (hept, $J=7.6 \mathrm{~Hz}, 3 \mathrm{H}), 1.12(\mathrm{~d}, J=7.6 \mathrm{~Hz}, 9 \mathrm{H}), 1.11(\mathrm{~d}, J=7.6 \mathrm{~Hz}, 9 \mathrm{H}) ;{ }^{13} \mathrm{C}$ NMR (125 MHz, CDCl $)$ d 173.7 (C), 149.3 (C), 136.6 (C), 134.9 (C), 133.8 (CH), 133.0 (C), $128.5(\mathrm{CH}), 127.72(\mathrm{CH}), 127.68(\mathrm{CH}), 127.5(\mathrm{CH}), 126.4(\mathrm{CH}), 126.3(\mathrm{CH}), 126.2$ $(\mathrm{CH}), 122.2(\mathrm{CH}), 104.7(\mathrm{CH}), 53.4(\mathrm{CH}), 51.7\left(\mathrm{CH}_{3}\right), 35.6(\mathrm{CH}), 31.1\left(\mathrm{CH}_{2}\right), 18.07$ $\left(\mathrm{CH}_{3}\right), 18.05\left(\mathrm{CH}_{3}\right), 12.7(\mathrm{CH})$; LRMS (ESI) $\mathrm{m} / z$ (relative intensity): $477.5\left([\mathrm{M}+\mathrm{H}]^{+}, 6\right)$, $499.3\left([\mathrm{M}+\mathrm{Na}]^{+}, 100\right)$; HRMS (ESI) $m / z$ Calcd for $\left[\mathrm{C}_{30} \mathrm{H}_{40} \mathrm{NaO}_{3} \mathrm{Si}^{+}\left([\mathrm{M}+\mathrm{Na}]^{+}\right)\right.$: 457.2169. Found: 457.2185. HPLC analysis: $91.3 \%$ ee (Chiralcel OD-H, $2.0 \% i-\mathrm{PrOH}$ in hexane, $0.8 \mathrm{~mL} / \mathrm{min}, \lambda=254 \mathrm{~nm}, \mathrm{t}_{\mathrm{R}}=6.0 \mathrm{~min}$, major; $\mathrm{t}_{\mathrm{R}}=8.9 \mathrm{~min}$, minor). 


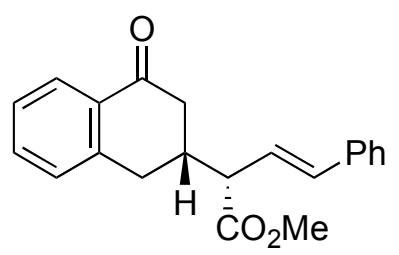

18

To a stirring solution of $\mathbf{1 7 b}(91 \mathrm{mg})$ in $\mathrm{CH}_{3} \mathrm{CN}(2 \mathrm{~mL})$ was added 3 drops of $\mathrm{HF}$ solution $(48 \%)$. The resulting solution was stirred for $1.5 \mathrm{~h}$ at $\mathrm{rt}$. Ethyl acetate $(30 \mathrm{~mL})$ was added. The mixture was washed with saturated $\mathrm{NaHCO}_{3}(5 \mathrm{~mL})$ and brine $(5 \mathrm{~mL})$, then dried over $\mathrm{MgSO}_{4}$. The solvent was evaporated under reduced pressure and the residue was purified by flash chromatography on silica gel (1:1 pentane/ether) to afford 18 (56 $\mathrm{mg}$, $84 \%$ yield).

(R, E)-methyl 2-((R)-1,2,3,4-tetrahydro-1-oxonaphthalen-3-yl)-4-phenylbut-3-enoate (18):

colorless oil; $\mathrm{R}_{\mathrm{f}} 0.40$ (1:1 pentane/ether); $[\alpha]_{\mathrm{D}}^{25}-66.1$ (c 3.20, $\mathrm{CHCl}_{3}$ ); FTIR (film): 3060, 3027, 2951, 2843, 1734, 1683, 1601, 1496, 1456, 1435, 1359, 1295, 1261, 1226, 1194, 1160, 1118, $1023 \mathrm{~cm}^{-1} ;{ }^{1} \mathrm{H}$ NMR $\left(500 \mathrm{MHz}, \mathrm{CDCl}_{3}\right) \delta 8.02(\mathrm{~d}, J=7.6 \mathrm{~Hz}, 1 \mathrm{H})$, $7.48(\mathrm{td}, J=7.3,1.2 \mathrm{~Hz}, 1 \mathrm{H}), 7.40(\mathrm{~d}, J=7.3 \mathrm{~Hz}, 2 \mathrm{H}), 7.36-7.30$ (m, 3H), 7.29-7.25 (m, $1 \mathrm{H}), 7.22(\mathrm{~d}, J=7.6 \mathrm{~Hz}, 1 \mathrm{H}), 6.52(\mathrm{~d}, J=15.9 \mathrm{~Hz}, 1 \mathrm{H}), 6.23(\mathrm{dd}, J=15.9,9.8 \mathrm{~Hz}, 1 \mathrm{H})$, 3.74 (s, 3H), 3.19 (appt. t, $J=8.8 \mathrm{~Hz}, 1 \mathrm{H}$ ), 3.07 (br d, $J=14.9 \mathrm{~Hz}, 1 \mathrm{H}$ ), 2.82-2.67 (m, 3H), 2.49 (dd, $J=16.4,11.2 \mathrm{~Hz}, 1 \mathrm{H}) ;{ }^{13} \mathrm{C}$ NMR $\left(125 \mathrm{MHz}, \mathrm{CDCl}_{3}\right) \delta$ 197.0, 172.8, 142.6, 136.2, 134.5, 133.8, 132.1, 129.0, 128.6, 128.0, 127.1, 127.0, 126.4, 124.6, 54.7, 52.1, 43.4, 37.5, 33.2; LC-MS (ESI) $\mathrm{m} / z$ (relative intensity): 319.2 ([M+H $]^{+}, 100$ ), 341.1 $\left([\mathrm{M}+\mathrm{Na}]^{+}, 0.4\right)$; HPLC analysis: $96.2 \%$ ee $(R, R$-Whelk O1, $20.0 \% i$-PrOH in hexane, 1.0 $\mathrm{mL} / \mathrm{min}, \lambda=254 \mathrm{~nm}, \mathrm{t}_{\mathrm{R}}=22.2$ min, major; $\mathrm{t}_{\mathrm{R}}=30.6$ min, minor); Anal. Calcd for $\mathrm{C}_{21} \mathrm{H}_{20} \mathrm{O}_{3}$ : C, 78.73; H, 6.29. Found: C, 78.65; H, 6.34.

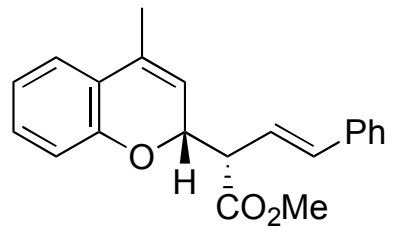


To a solution of 4-methyl-2H-cromene $19(0.5 \mathrm{mmol})$ and $\mathrm{Rh}_{2}(S \text {-DOSP })_{4}(4.8 \mathrm{mg}, 0.0025$ mmol) in 2,2-dimethylbutane $(1 \mathrm{~mL})$ was added a solution of (E)-methyl phenylvinyldiazoacetate $1(111 \mathrm{mg}, 0.55 \mathrm{mmol})$ in DMB $(5 \mathrm{~mL})$ over a 45 min period via syringe-pump at $0{ }^{\circ} \mathrm{C}$ (with crashed-ice cooling). The resulting mixture was then stirred for another $5 \mathrm{~min}$. The solvent was then evaporated under reduced pressure and the residue was purified by flash chromatography on silica gel (10:1 pentane/ether eluent) to provide 20 (120 $\mathrm{mg}, 75 \%$ yield).

\section{$(S, 3 E)$-methyl 2-((R)-4-methyl-2H-chromen-2-yl)-4-phenylbut-3-enoate (20):}

colorless oil; $\mathrm{R}_{\mathrm{f}} 0.35$ (5:1 pentane/ether); $[\alpha]_{\mathrm{D}}{ }^{25}+25.1$ (c 3.38, $\mathrm{CHCl}_{3}$ ); FTIR (film): 3028, 2950, 1734, 1654, 1605, 1576, 1488, 1450, 1435, 1380, 1356, 1226, 1164, 1128, 1041, $1010 \mathrm{~cm}^{-1} ;{ }^{1} \mathrm{H}$ NMR $\left(500 \mathrm{MHz}, \mathrm{CDCl}_{3}\right) \delta$ 7.30-7.25 (m, 4H), 7.24-7.19 (m, 1H), 7.15-7.10 (m, 2H), 6.90 (t, $J=7.5 \mathrm{~Hz}, 1 \mathrm{H}), 6.79(\mathrm{~d}, J=8.2 \mathrm{~Hz}, 1 \mathrm{H}), 6.42$ (d, $J=15.9 \mathrm{~Hz}$, $1 \mathrm{H}), 6.26$ (dd, $J=15.9,9.1 \mathrm{~Hz}, 1 \mathrm{H}), 5.53$ (br d, $J=2.4 \mathrm{~Hz}, 1 \mathrm{H}), 5.19$ (m, 1H), 3.70 (s, $3 \mathrm{H}), 3.52(\mathrm{dd}, J=9.1,7.2 \mathrm{~Hz}, 1 \mathrm{H}), 2.01(\mathrm{~s}, 3 \mathrm{H}) ;{ }^{13} \mathrm{C} \mathrm{NMR}\left(125 \mathrm{MHz}, \mathrm{CDCl}_{3}\right) \delta 171.6$, 152.6, 136.6, 134.1 (2C), 131.2, 129.3, 128.4, 127.6, 126.4, 123.5, 123.2, 121.1, 119.1, 116.2, 75.1, 54.8, 52.1, 18.0; LC-MS (ESI) $\mathrm{m} / z$ (relative intensity): 241.7 (82), 268.2 (30), 294.2 (37), $321\left([\mathrm{M}+\mathrm{H}]^{+}, 100\right)$; HPLC analysis: 95.1\% ee (Chiralcel OD-H, 2.0\% $i$ $\mathrm{PrOH}$ in hexane, $0.8 \mathrm{~mL} / \mathrm{min}, \lambda=254 \mathrm{~nm}, \mathrm{t}_{\mathrm{R}}=11.9 \mathrm{~min}$, minor; $\mathrm{t}_{\mathrm{R}}=39.2 \mathrm{~min}$, major); Anal. Calcd for $\mathrm{C}_{21} \mathrm{H}_{20} \mathrm{O}_{3}$ : C, 78.73; H, 6.29. Found: C, 78.63; H, 6.29.

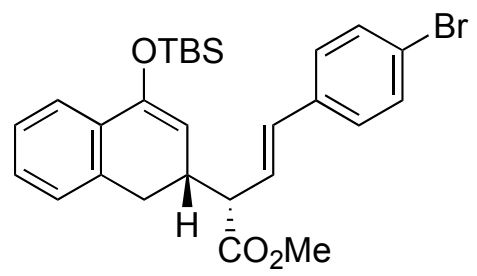

A

To a solution of tert-butyl-(3,4-dihydronaphthalen-1-yloxy)-dimethylsilane $\mathbf{1 6 b}(0.5$ $\mathrm{mmol})$ and $\mathrm{Rh}_{2}(S \text {-DOSP })_{4}(4.8 \mathrm{mg}, 0.0025 \mathrm{mmol})$ in 2,2-dimethylbutane (1 mL) was added a solution of $(E)$-methyl $p$-bromophenylvinyldiazoacetate $(155 \mathrm{mg}, 0.55 \mathrm{mmol})$ in $\operatorname{DMB}(9 \mathrm{~mL})$ over a $45 \mathrm{~min}$ period via syringe-pump at $0{ }^{\circ} \mathrm{C}$ (with crashed-ice cooling). 
The resulting mixture was then stirred for another $4 \mathrm{~h}$ at $\mathrm{rt}$. The solvent was then evaporated under reduced pressure and the residue was purified by flash chromatography on silica gel (20:1 pentane/ether eluent) to provide A (173 mg, 67\% yield).

\section{$(S, 3 E)$-methyl 4-(4-bromophenyl)-2-((R)-1,2-dihydro-4-tert-}

\section{butyldimethylsiloxylnaphthalen-2-yl)but-3-enoate (A):}

colorless oil; $\mathrm{R}_{\mathrm{f}} 0.33$ (10:1 pentane/ether); $[\alpha]_{\mathrm{D}}{ }^{25}-75.5$ (c 2.69, $\mathrm{CHCl}_{3}$ ); FTIR (film): 3024, 2952, 2930, 2885, 2857, 1734, 1635, 1487, 1471, 1434, 1363, 1341, 1257, 1158, 1072, $1009 \mathrm{~cm}^{-1} ;{ }^{1} \mathrm{H}$ NMR $\left(500 \mathrm{MHz}, \mathrm{CDCl}_{3}\right) \delta 7.47(\mathrm{~d}, J=7.3 \mathrm{~Hz}, 1 \mathrm{H}), 7.42(\mathrm{~d}, J=8.5$ $\mathrm{Hz}, 2 \mathrm{H}), 7.23-7.15$ (m, 2H), 7.17 (d, $J=8.5 \mathrm{~Hz}, 2 \mathrm{H}), 7.05$ (d, $J=7.0 \mathrm{~Hz}, 1 \mathrm{H}), 6.32(\mathrm{~d}, J$ $=15.9 \mathrm{~Hz}, 1 \mathrm{H}), 6.13(\mathrm{dd}, J=15.9,9.6 \mathrm{~Hz}, 1 \mathrm{H}), 5.09(\mathrm{~d}, J=5.2 \mathrm{~Hz}, 1 \mathrm{H}), 3.68(\mathrm{~s}, 3 \mathrm{H})$, 3.09 (appt. t, $J=9.6 \mathrm{~Hz}, 1 \mathrm{H}), 2.94-2.85$ (m, 2H), 2.77-2.70 (m, 1H), 1.01 (s, 9H), 0.21 (s, $3 \mathrm{H}), 0.19(\mathrm{~s}, 3 \mathrm{H}) ;{ }^{13} \mathrm{C} \mathrm{NMR}\left(125 \mathrm{MHz}, \mathrm{CDCl}_{3}\right) \delta$ 173.5, 149.3, 135.5, 134.9, 133.0, 132.5, 131.6, 127.9, 127.8, 127.5, 126.9, 126.4, 122.2, 121.4, 105.5, 53.4, 51.8, 35.6, 31.0, 25.8, 18.3, -4.4, -4.8; LRMS (ESI) $\mathrm{m} / z$ (relative intensity): 398.9 (52), 513.0 ([M+Na $\left.]^{+}, 98\right)$; HRMS (ESI) $m / z$ Calcd for $\left[\mathrm{C}_{27} \mathrm{H}_{33} \mathrm{BrNaO}_{3} \mathrm{Si}\right]^{+}\left([\mathrm{M}+\mathrm{Na}]^{+}\right)$: 535.1275 . Found: 535.1288. HPLC analysis: $95.2 \%$ ee (Chiralcel OD-H, $0.5 \% i$-PrOH in hexane, $0.8 \mathrm{~mL} / \mathrm{min}, \lambda=254 \mathrm{~nm}, \mathrm{t}_{\mathrm{R}}=11.1 \mathrm{~min}$, minor; $\mathrm{t}_{\mathrm{R}}=13.6 \mathrm{~min}$, major).

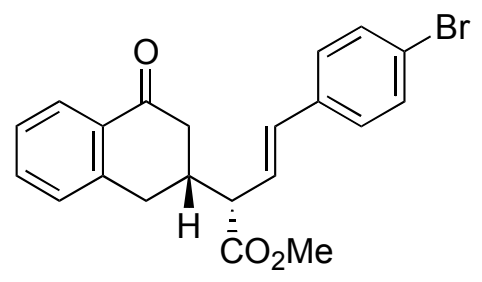

B

To a stirring solution of $\mathbf{A}(102 \mathrm{mg})$ in $\mathrm{CH}_{3} \mathrm{CN}(5 \mathrm{~mL})$ was added 4 drops of $\mathrm{HF}$ solution (48\%). The resulting solution was stirred for $1 \mathrm{~h}$ at $\mathrm{rt}$. The mixture was diluted with Ethyl acetate $(30 \mathrm{~mL})$, washed with saturated $\mathrm{NaHCO}_{3}(5 \mathrm{~mL})$ and brine $(5 \mathrm{~mL})$, then dried over $\mathrm{MgSO}_{4}$. The solvent was evaporated under reduced pressure and the residue was purified by flash chromatography on silica gel (1:1 pentane/ether) to afford $\mathbf{B}(67 \mathrm{mg}$, $83 \%$ yield) as a white solid which can be recrystalized from ether/pentane to give a colorless crystal $(98.6 \%$ ee) suitable for X-ray crystallography. 


\section{$(R, E)-m e t h y l$ 4-(4-bromophenyl)-2-((R)-1,2,3,4-tetrahydro-1-oxonaphthalen-3-yl) but -3-enoate (B):}

white solid, mp 103-112 ${ }^{\circ} \mathrm{C} ; \mathrm{R}_{\mathrm{f}} 0.35$ (1:1 pentane/ether); $[\alpha]_{\mathrm{D}}{ }^{25}-65.9$ (c 3.35, $\mathrm{CHCl}_{3}$ ); FTIR $\left(\mathrm{CH}_{2} \mathrm{Cl}_{2}\right)$ : 3027, 2949, 1733, 1684, 1603, 1487, 1455, 1434, 1295, 1259, 1227 , 1194, 1160, 1118, 1073, $1008 \mathrm{~cm}^{-1} ;{ }^{1} \mathrm{H}$ NMR (500 MHz, $\left.\mathrm{CDCl}_{3}\right) \delta 8.02(\mathrm{dd}, J=7.6,0.9$ $\mathrm{Hz}, 1 \mathrm{H}), 7.48(\mathrm{td}, J=7.6,0.9 \mathrm{~Hz}, 1 \mathrm{H}), 7.45(\mathrm{~d}, J=8.5 \mathrm{~Hz}, 2 \mathrm{H}), 7.32(\mathrm{t}, J=7.6 \mathrm{~Hz}, 1 \mathrm{H})$, $7.25(\mathrm{~d}, J=8.5 \mathrm{~Hz}, 2 \mathrm{H}), 7.22(\mathrm{~d}, J=7.6 \mathrm{~Hz}, 1 \mathrm{H}), 6.45$ (d, $J=15.9 \mathrm{~Hz}, 1 \mathrm{H}), 6.23$ (dd, $J=$ 15.9, 9.7 Hz, 1H), 3.74 (s, 3H), 3.18 (appt. t, $J=9.5 \mathrm{~Hz}, 1 \mathrm{H}), 3.04$ (br d, $J=15.0 \mathrm{~Hz}$, 1H), 2.82-2.67 (m, 3H), $2.48(\mathrm{dd}, J=16.4,11.5 \mathrm{~Hz}, 1 \mathrm{H}) ;{ }^{13} \mathrm{C} \mathrm{NMR}\left(125 \mathrm{MHz}, \mathrm{CDCl}_{3}\right) \delta$ $196.9,172.6,142.4,135.1,133.8,133.3,132.1,131.7,129.0,128.0,127.1,127.0,125.4$, 121.8, 54.6, 52.2, 43.3, 37.5, 33.2; LRMS (ESI) $m / z$ (relative intensity): $399.1\left([\mathrm{M}+\mathrm{H}]^{+}\right.$, 9), $421.0\left([\mathrm{M}+\mathrm{Na}]^{+}, 95\right)$; HRMS (ESI) $m / z$ Calcd for $\left[\mathrm{C}_{21} \mathrm{H}_{19} \mathrm{BrNaO}_{3}\right]^{+}\left([\mathrm{M}+\mathrm{Na}]^{+}\right)$: 421.0410. Found: 421.0402. HPLC analysis: 98.6\% ee (Chiralcel OD-H, 2.0\% $i$-PrOH in hexane, $0.8 \mathrm{~mL} / \mathrm{min}, \lambda=254 \mathrm{~nm}, \mathrm{t}_{\mathrm{R}}=35.6 \mathrm{~min}$, minor; $\mathrm{t}_{\mathrm{R}}=37.7 \mathrm{~min}$, major). Anal. Calcd for $\mathrm{C}_{21} \mathrm{H}_{19} \mathrm{BrO}_{3}$ : C, 63.17; H, 4.80; Br, 20.01. Found: C, 63.23; H, 4.79; Br, 19.77.

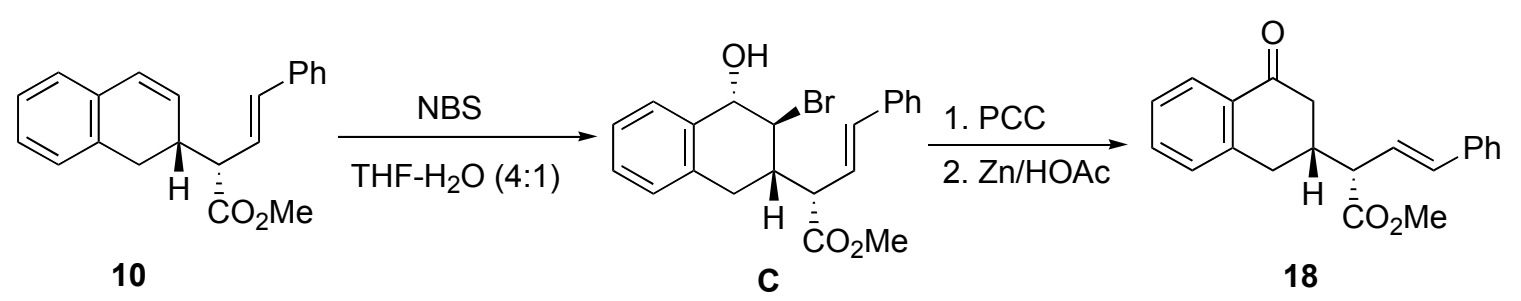

In order to prove the stereochemistry, $\mathbf{1 0}$ was converted to $\mathbf{1 8}$ by the following procedure (conditions have not been optimized):

To a solution of $10(50 \mathrm{mg})$ in THF- $\mathrm{H}_{2} \mathrm{O}(4: 1,2.25 \mathrm{~mL})$ was added NBS $(32.5 \mathrm{mg}, 1.1$ eq.) at rt. The resulting mixture was stirred for $0.5 \mathrm{~h}$, then diluted with EtOAc $(20 \mathrm{~mL})$, washed with brine $(10 \mathrm{~mL})$ and dried over $\mathrm{MgSO}_{4}$. The crude product was purified by flash chromatography on silica gel (1:1 pentane/ether eluent) to give C (54 mg, 81\% yield). ${ }^{1} \mathrm{H}$ NMR (500 MHz, $\left.\mathrm{CDCl}_{3}\right) \delta$ 7.43-7.40 (m, 2H), 7.37-7.32 (m, 3H), 7.30-7.22 (m, 3H), 7.11-7.07 (m, 1H), $6.66(\mathrm{~d}, J=15.9 \mathrm{~Hz}, 1 \mathrm{H}), 6.21(\mathrm{dd}, J=15.9,9.8 \mathrm{~Hz}, 1 \mathrm{H})$, $4.98(\mathrm{~d}, J=2.7 \mathrm{~Hz}, 1 \mathrm{H}), 4.44(\mathrm{~s}, 1 \mathrm{H}) 3.76(\mathrm{~s}, 3 \mathrm{H}), 3.43$ (appt. t, $J=9.9 \mathrm{~Hz}, 1 \mathrm{H}), 2.86-$ 
$2.76(\mathrm{~m}, 2 \mathrm{H}), 2.72-2.62(\mathrm{~m}, 1 \mathrm{H}), 2.53$ (br s, $1 \mathrm{H}) ;{ }^{13} \mathrm{C} \mathrm{NMR}\left(125 \mathrm{MHz}, \mathrm{CDCl}_{3}\right) \delta$ 173.0, $136.2,134.8,134.6,133.6,130.5,129.0,128.65,128.57,128.0,126.8,126.5,124.7,72.9$, $55.8,53.8,52.3,35.0,29.4$.

Pyridinium chlorochromate (54 mg, 2 eq.) was added to a stirring suspension of $\mathbf{C}$ (45 $\mathrm{mg}$ ), NaOAc (20 mg, 2 eq.) and celite in DCM $(5 \mathrm{~mL})$ at rt. The mixture was stirred for $3 \mathrm{~h}$ at $\mathrm{rt}$ and then filtrated through a pad of silica gel. The cake was washed with DCM and ether. The solvent was removed and the crude product was purified by flash chromatography on silica gel (2:1 pentane/ether eluent) to afford the ketone $(33.5 \mathrm{mg}$, $74 \%$ yield). ${ }^{1} \mathrm{H}$ NMR (500 MHz, $\left.\mathrm{CDCl}_{3}\right) \delta 8.09$ (d, $\left.J=8.0 \mathrm{~Hz}, 1 \mathrm{H}\right), 7.51(\mathrm{t}, J=7.3 \mathrm{~Hz}$, 1H), 7.43 (d, $J=7.6 \mathrm{~Hz}, 2 \mathrm{H}), 7.38-7.33(\mathrm{~m}, 3 \mathrm{H}), 7.32-7.27$ (m, 1H), 7.23 (d, $J=7.9 \mathrm{~Hz}$, 1H), 6.70 (d, $J=15.9 \mathrm{~Hz}, 1 \mathrm{H}), 6.16$ (dd, $J=15.9,9.8 \mathrm{~Hz}, 1 \mathrm{H}), 4.24$ (br d, $J=2.1 \mathrm{~Hz}$, 1H), 3.77 (s, 3H), 3.52 (appt. t, $J=9.8 \mathrm{~Hz}, 1 \mathrm{H}$ ), 2.96-2.86 (m, 2H), 2.67-2.61 (m, 1H); ${ }^{13} \mathrm{C}$ NMR $\left(125 \mathrm{MHz}, \mathrm{CDCl}_{3}\right) \delta 190.0,172.1,141.7,135.9,135.5,134.3,129.1,128.9$, $128.7,128.2,127.3,126.5,123.7,54.1,53.1,52.4,40.7,29.3$.

To a stirring solution of the ketone obtained the above step $(14 \mathrm{mg})$ in HOAc $(2 \mathrm{~mL})$ was added zinc dust ( $12 \mathrm{mg}, 5$ eq.) at rt. The mixture was stirred overnight, diluted with EtOAc $(20 \mathrm{~mL})$, washed with $\mathrm{NaHCO}_{3}$ and brine, and then dried over $\mathrm{MgSO}_{4}$. The crude product was purified by flash chromatography (2:1 petroleum ether/ether eluent) to give 18 (6.5 mg, 56\% yield). The ${ }^{1} \mathrm{H}$ NMR data, HPLC trace ( $>99 \%$ ee) and optical rotation sign $\left([\alpha]_{\mathrm{D}}^{22}-56.0\left(c 0.65, \mathrm{CHCl}_{3}\right)\right)$ of $\mathbf{1 8}$ were consistent with the data of $\mathbf{1 8}$ obtained from 16. 
X-ray Structure of compound $\mathbf{B}:{ }^{9}$<smiles>COC(=O)[C@H]1CC(=O)c2ccccc2C1</smiles><smiles>C#CC(=O)[C@H](/C=C/c1ccc(Br)cc1)[C@H]1CC(=O)c2ccccc2C1</smiles>

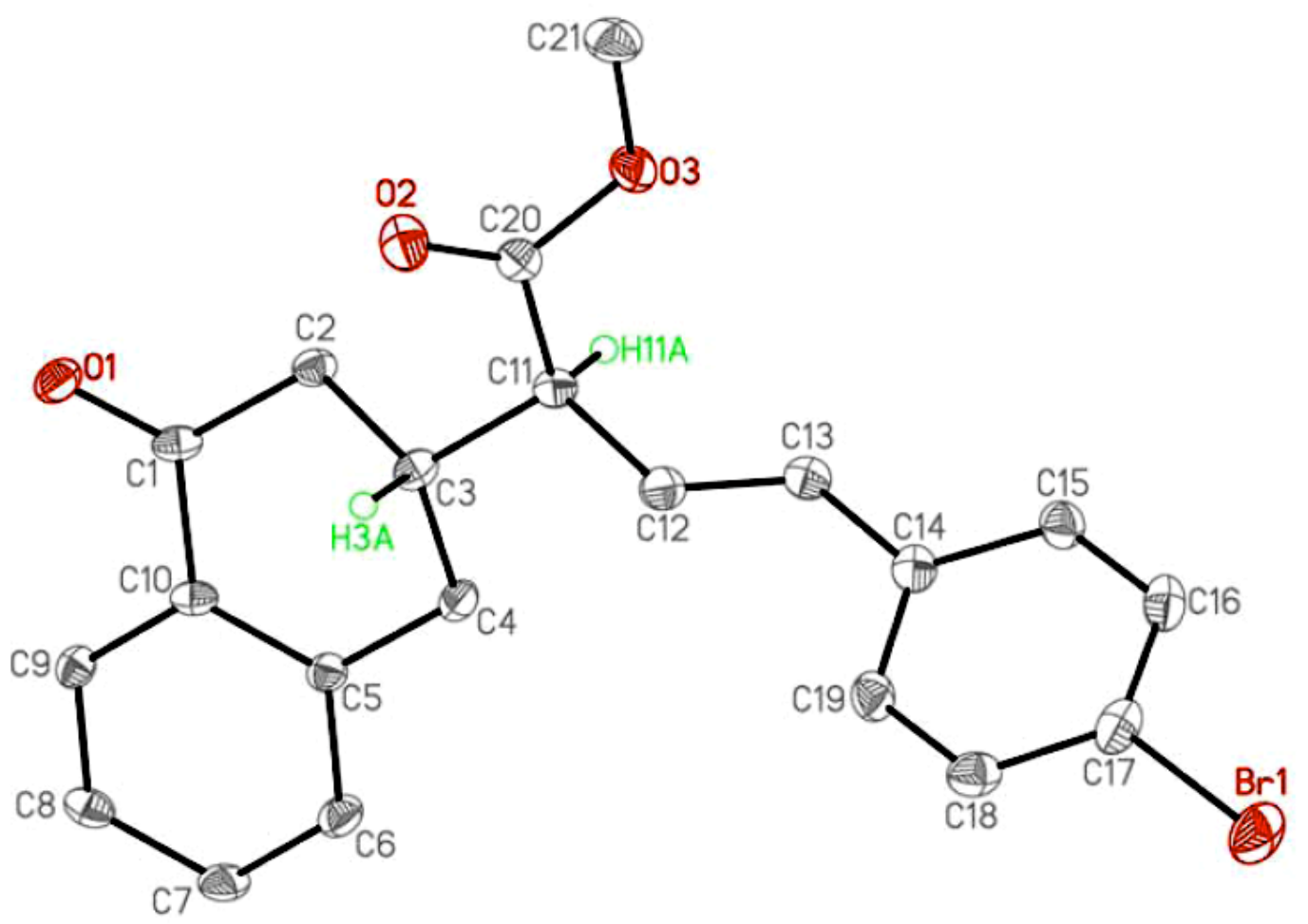

Torsion angle $\mathrm{C} 3-\mathrm{C} 11-\mathrm{C} 20-\mathrm{O} 2=17.8(4)^{\circ}$

\footnotetext{
${ }^{9}$ The X-ray crystallographic data have been submitted to the Cambridge Structure Database: Gerlits, O. O. and Coppens, P. Private Communication (1078), 2004, CCDC 237040.
} 


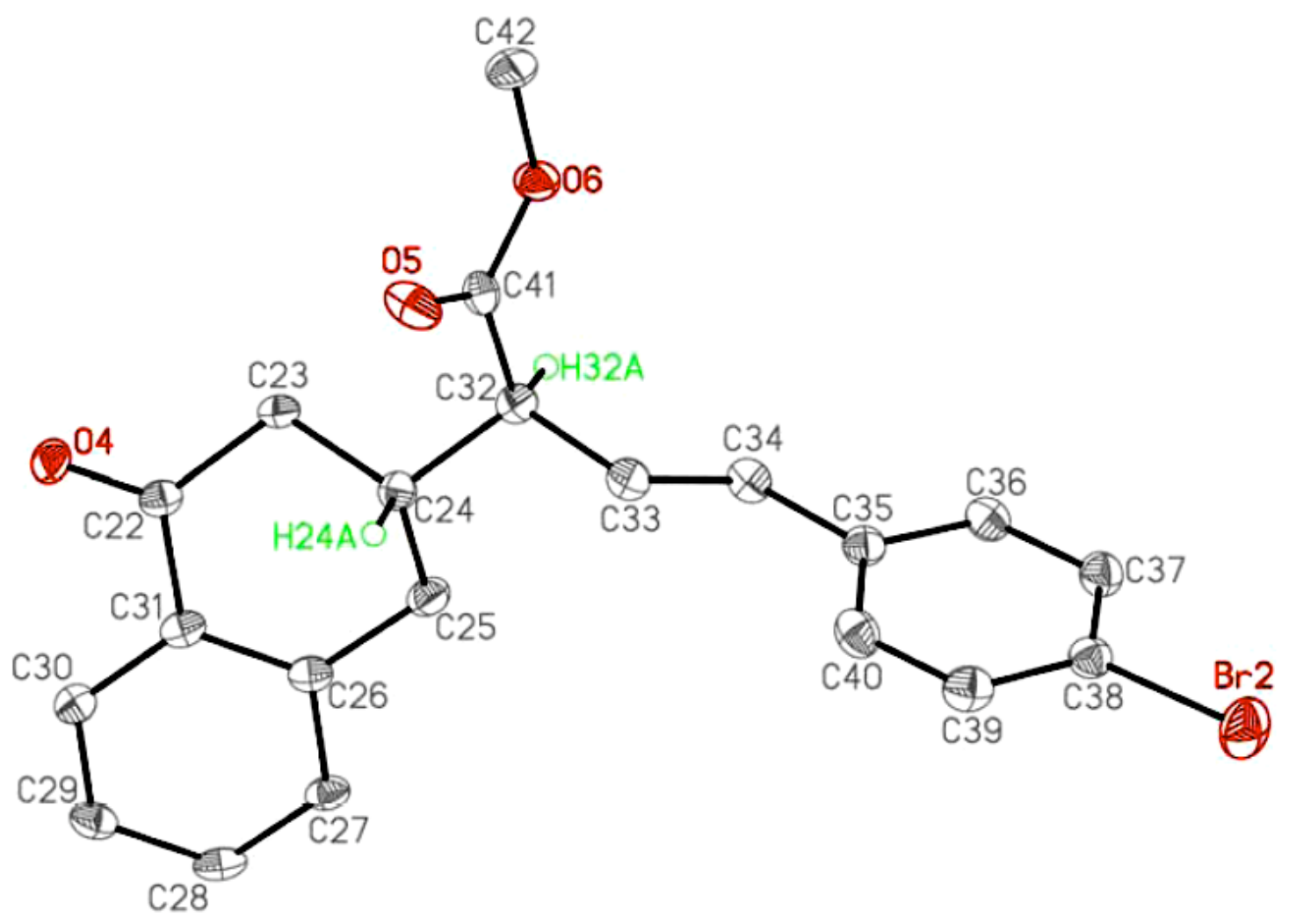

Torsion angle $\mathrm{C} 24-\mathrm{C} 32-\mathrm{C} 41-\mathrm{O} 5=40.8(4)^{\circ}$ 